Cahiers $d u$ MONDE RUSSE

\section{Cahiers du monde russe}

Russie - Empire russe - Union soviétique et États indépendants

$50 / 2-3 \mid 2009$

L'Europe orientale, 1650-1730. Crises, conflits et renouveau

\title{
John Hebdon à Moscou (1677-1678)
}

Histoire d'une crise diplomatique

John Hebdon in Moscow (1677-1678). The story of a diplomatic crisis

\section{Sergej P. Orlenko}

\section{(2) OpenEdition}

\section{Journals}

Édition électronique

URL : https://journals.openedition.org/monderusse/9722

DOI : 10.4000/monderusse. 9722

ISSN : $1777-5388$

Éditeur

Éditions de l'EHESS

Édition imprimée

Date de publication : 15 septembre 2009

Pagination : 441-451

ISBN : 978-2-7132-2260-3

ISSN : $1252-6576$

Référence électronique

Sergej P. Orlenko, « John Hebdon à Moscou (1677-1678) », Cahiers du monde russe [En ligne], 50/2-3 | 2009, mis en ligne le 13 octobre 2012, consulté le 04 septembre 2022. URL : http://

journals.openedition.org/monderusse/9722 ; DOI : https://doi.org/10.4000/monderusse.9722 


\section{JOHN HEBDON À MOSCOU (1677-1678)}

\section{Histoire d'une crise diplomatique}

Les historiens connaissent bien le nom de John Hebdon père. Homme de confiance du gouvernement russe, il remplissait diverses missions hors des frontières pour les autorités de Moscou : il vendait à l'étranger les marchandises dont le Trésor avait le monopole, y achetait des armes pour l'armée russe, des objets de luxe à l'usage de la cour, y enrôlait des « hommes d'armes » au service de la Russie et il contribua à la capture du «criminel et imposteur Timoška Ankudinov » ${ }^{1}$. Royaliste convaincu, Hebdon travailla activement, après la restauration de la monarchie en GrandeBretagne, à la reprise des relations diplomatiques anglo-russes à un haut niveau. Ses mérites étaient également très appréciés du roi d'Angleterre : c'est ainsi que Charles II conféra à Hebdon père les titres de chevalier [knight] et de valet de chambre du roi (groom of the Chamber). En 1667, Hebdon père se rend en Russie en qualité cette fois d'envoyé ${ }^{2}$ extraordinaire de la couronne britannique. Dans ses lettres de créance, Charles II le recommande au tsar Aleksej Mihajlovič en ces termes : « notre fidèle et aimé serviteur et $[. .$.$] valet de chambre de Notre Royale Majesté »^{3}$.

1. Cf. Il'ja Jakovlevič Gurljand, Ivan Gebdon: Komisarius i rezident [Ivan Hebdon, chargé d'affaires et résident], Jaroslavl', 1903 ; Jan Willem Veluwenkamp, Arhangel'sk: Niderlandskie predprinimateli $v$ Rossii 1550-1785 [Arkangel'sk : Les entrepreneurs hollandais en Russie, 1550-1785], M. : ROSSPEN, 2006, p. 149, 151, 152.

2. Les Russes distinguaient, selon un ordre hiérarchique décroissant correspondant à la nomenclature en usage dans le reste de l'Europe, les ambassadeurs (sing. posol), les envoyés (sing. poslannik), les courriers (sing. gonec) (NdT).

3. RGADA, f. 35, «Snošenija s Angliej » [Relations avec l'Angleterre], 1667, n 218, « Priezd v Moskvu anglijskogo črezvyčajnogo poslannika Ivana Gebdona s uvedomleniem protivnyh Anglii postupkah gollandcev...» [Arrivée à Moscou de l'envoyé extraordinaire anglais Ivan Hebdon, avec des informations concernant certaines actions commises par les Hollandais portant préjudice à l'Angleterre... ], 1. 95. 
Son fils et homonyme parfait, John Hebdon (fils), passa sa jeunesse en Moscovie. En Europe, il aida son père à recruter des officiers et convoya en Russie d'importantes quantités d'armes achetées en Hollande. Cependant, après la restauration des Stuarts, John Hebdon fils, tout comme son père, se consacre entièrement au service de la couronne britannique. La connaissance qu'il a de la Russie l'aide du reste dans sa carrière : ainsi, dix ans après son père, en 1677, il se rend à Moscou en qualité d'envoyé extraordinaire du monarque anglais ${ }^{4}$.

L'histoire de Hebdon ne fait pas figure d'exception. Dans la seconde moitié du $\mathrm{XVII}^{\mathrm{e}}$ siècle en Angleterre, mais aussi dans d'autres pays européens, les gouvernements comprirent l'avantage qu'il y avait à envoyer en Moscovie des diplomates choisis parmi des personnes plus ou moins familiarisées avec les réalités russes, qui étaient loin d'être simples (ils étaient en majorité originaires de familles de marchands). C'est ainsi que Kunraad van Klenk, d'une ancienne famille de marchands présente en Russie depuis plusieurs générations, est nommé résident des Provinces-Unies. La couronne danoise, elle, était représentée par l'entrepreneur et manufacturier célèbre en Moscovie, Heinrich [Andrej Ivanovič] Butenant von Rosenbusch.

L'essentiel de la mission diplomatique de John Hebdon fils se résumait en deux points. Tout d'abord, il était chargé de féliciter le tsar Fëdor à l'occasion de son avènement et, en second lieu, devait réclamer le rétablissement des privilèges commerciaux de la Compagnie de Moscovie, requête devenue traditionnelle pour les diplomates britanniques. Hebdon transmit les félicitations du roi, mais essuya un refus, tout aussi traditionnel, concernant les privilèges. En effet, la tendance au protectionnisme répandue dans toute l'Europe n'épargnait pas la Russie, et le nouveau règlement de Commerce (Novotorgovyj ustav), adopté en 1667, en est la parfaite illustration.

Malgré son échec et l'impossibilité manifeste d'y remédier, John Hebdon ne se hâtait pas de quitter la capitale russe et, plus de six mois après son arrivée à Moscou, le secrétariat des Ambassades (Posol'skij prikaz) finit par lui demander avec insistance : « pourquoi ne retournait-il pas auprès de Sa Royale Majesté ? ». Hebdon invoqua l'absence d'un ordre du roi en ce sens et les nombreuses affaires d'ordre personnel qu'il lui restait à résoudre. Quelque temps après, il fut décidé que l'envoyé, qui vivait à Moscou sans être chargé d'aucune mission, serait privé de « vivres » (korm), c'est-à-dire de la prestation de subsistance en nature accordée par la Russie aux diplomates étrangers. En réponse aux protestations de Hebdon, le secrétariat des Ambassades déclara que, étant donné qu'une réponse aux lettres du roi avait été donnée à l'envoyé, « le protocole diplomatique avait été observé ». Si cependant Hebdon ne quittait pas Moscou, il devrait vivre « à ses frais » : « ce séjour est contraire à l'usage des ambassades, jamais on n'a fourni de vivres à quelqu'un sans mission $»^{5}$. Mais cela ne suffit pas à faire partir Hebdon. Visiblement, quelque

4. Voir Gurljand, Ivan Gebdon: Komisarius i rezident.

5. RGADA, f. 35, 1676, n 234, « Priezd v Moskvu i otpusk črezvyčajnogo poslannika Ivana Gebdona... » [Arrivée à Moscou et audience de congé de l'envoyé extraordinaire anglais Ivan Hebdon], 1. 328-332. 
chose le retenait à Moscou, bien que sa vie dans la capitale russe fût loin d'être agréable ou même tranquille.

Le séjour de John Hebdon prit en effet un caractère particulièrement scandaleux et fut marqué par de nombreux conflits et des querelles entre l'envoyé, secondé par ses gens, et divers personnages, aussi bien des Russes que des étrangers ${ }^{6}$. Entre Hebdon et une partie des officiers étrangers en service dans l'armée russe s'établit ainsi une inimitié particulièrement vive, qui se transforma avec le temps en une haine sanglante.

La raison de ce conflit ne manque pas d'originalité. John Hebdon père, lorsqu'il était au service de la Russie, recrutait à l'étranger des officiers pour l'armée russe. Lorsqu'il passait contrat avec ces militaires, Hebdon leur donnait de la main à la main de l'argent pour leur voyage. Bien que cet argent lui fût alloué par le Trésor russe, Hebdon leur faisait signer les reçus à son nom. Arrivés en Russie, les officiers rejoignaient leur régiment ; le remboursement de l'avance qu'ils avaient reçue était échelonné : le Trésor en retenait chaque mois une partie sur leur traitement, et le secrétariat des Étrangers (Inozemnyj prikaz) leur délivrait des attestations à cet effet. Et voilà que soudain, quelques années plus tard, Hebdon fils arrive en Russie et présente aux officiers leurs billets à ordre en exigeant un paiement immédiat ! Les officiers refusèrent catégoriquement de rembourser leur dette une seconde fois. Hebdon insista et le conflit s'envenima. Menaces et insultes se mirent à fuser. Les supérieurs hiérarchiques ainsi que les compagnons d'armes des officiers prirent leur défense et une proportion importante des militaires étrangers qui vivaient à Moscou finit par se trouver impliquée dans la querelle avec le diplomate.

Le temps passant, leurs relations se détériorèrent jusqu'au jour où le sang finit par couler. Une querelle éclata en pleine rue : quatre serviteurs de Hebdon attaquèrent au sabre le capitaine Laurent von der Bukom (von Olden) [peut-être : Bockhoven] et le blessèrent gravement. Le lieutenant-colonel Daniel Zoege von Manteuffel [Danilo Cej] réussit à éviter la mort à son camarade en braquant ses pistolets sur les Anglais, puis il se réfugia, avec le blessé qui perdait son sang, dans la maison du colonel Martin Gulik [peut-être : Gulitz], aussitôt assiégé par les agresseurs. Bientôt les Anglais reçurent des renforts, avec à leur tête Hebdon en

\footnotetext{
6. RGADA, f. 141 (PDSL), 1678, $n^{\circ}$ 154, « Delo o smertnom ubijstve rotmistra Lavrenija fon-den Bukoma tako že o boju dvorjanina Ivana Pazuhina i karaul'nyh strel'cov v Nemeckoj slobode ... » [Affaire de l'agression meurtrière contre le commandant de compagnie Laurent von den Bukom [peut-être : Bockhoven] et des coups donnés au gentilhomme Ivan Pazuhin et aux mousquetaires du guet dans le faubourg des Étrangers], 1. 1-2.

7. La restitution des noms étrangers rencontrés dans un texte russe du XVII ${ }^{\mathrm{e}}$ siècle est souvent problématique. En l'absence de signatures, d'épitaphes ou de documents authentiques, écrits dans la langue des intéressés, les transcriptions, parfois fantaisistes et toujours approximatives, de l'administration moscovite ne permettent pas de déduire avec certitude leur orthographe véritable, et cela d'autant plus que dans l'Europe moderne, celle-ci n'était pas encore fixée. L'article de Erik Amburger, « Die weiteren Schicksale der alten Einwohnerschaft der Moskauer Ausländer-Sloboda seit der Zeit Peters I », Jahrbücher für Geschichte Osteuropas, NF 20, 1972, p. 412-426, repris dans Fremde und Einheimische im Wirtschafts- und Kulturleben des neuzeitlichen Russland, Wiesbaden : Franz Steiner, 1982 (Quellen und Studien zur Geschichte des Östlichen Europa, XVII), du même auteur, p. 275-289, nous a été d'un grand secours (NdT).
} 
personne. Seule l'intervention du propriétaire, qui menaça d'abattre le premier qui oserait franchir le seuil de sa maison, les dissuada de donner l'assaut ${ }^{8}$.

Le lendemain, l'envoyé, prenant conscience de l'ampleur des événements, éprouva de vives inquiétudes. Les militaires étrangers qui venaient servir dans l'armée russe étaient loin d'être des enfants de chœur et avaient généralement déjà combattu comme mercenaires pour d'autres pays d'Europe. Ils étaient soudés par l'esprit de corps et n'étaient pas habitués à souffrir d'offense ou d'affront sans réagir. Le 31 décembre 1677, Hebdon écrivit une lettre au secrétariat des Ambassades, dans laquelle il informait les autorités que certains officiers se réunissaient la nuit chez le colonel Heinrich [Andrej] Zoege, et complotaient de l'assassiner, lui et ses gens. Hebdon exigeait la protection du gouvernement russe, soulignant qu'il était du devoir des autorités de protéger, non seulement la vie, mais aussi l'honneur et la dignité des diplomates étrangers. Pour montrer avec quel soin l'administration anglaise protégeait la dignité des Russes, il citait un épisode curieux. «Alors que des ambassadeurs de Votre [...] Majesté se trouvaient en Angleterre, l'un de vos gentilshommes se promenait dans une rue où de jeunes enfants jouaient avec une balle en cuir. L'un d'eux frappa du pied la balle qui atteignit le gentilhomme. Une plainte fut adressée à Sa Royale Majesté. Sa Royale Majesté, ne doutant pas de la véracité des faits, donna l'ordre de mettre la corde au cou de ce jeune garçon et de le livrer ainsi à l'ambassadeur de Votre Majesté. Mais les ambassadeurs pardonnèrent au jeune garçon, car ils ne souhaitaient pas sa mort. C'est un exemple de l'amour fraternel que porte Sa Royale Majesté à Votre Majesté »9 .

Nous ne savons pas dans quelle mesure cette dénonciation était véridique. Les officiers avaient-ils réellement l'intention de régler son compte à Hebdon ou bien ce dernier prenait-il les devants à titre préventif ? Malgré la menace d'une vengeance des militaires, Hebdon se rendait périodiquement en ville, accompagné d'une escorte armée, et visitait régulièrement le faubourg des Étrangers (Nemeckaja sloboda). L'une de ces visites connut un dénouement tragique. Le 14 janvier 1678, peu après une heure du matin, un Russe, accompagné d'un des gens de Hebdon, sortit de chez le marchand étranger Pieter Hassenius, un résident du faubourg des Étrangers, chez qui Hebdon était invité ce soir-là. Dans la grand'rue, face à la maison du colonel Alexander Hamilton, ils tombèrent nez à nez avec une patrouille du guet, composée de mousquetaires (strel'cy) et commandée par Ivan Pazuhin (gentilhomme officiellement responsable de la police dans le faubourg des Étrangers). Sur l'ordre de ce dernier, le Russe fut capturé par les mousquetaires et l'étranger fit demi-tour. Le guet, avec son prisonnier, ne s'était pas beaucoup éloigné du lieu de l'incident lorsqu'il fut subitement attaqué par des hommes armés et, bien que les assaillants fussent deux fois moins nombreux que les mousquetaires, ces derniers eurent fort à faire. Les mousquetaires avaient en effet laissé leurs armes au siège de l'administration ( $s^{\prime \prime}$ ezžaja izba) et patrouillaient dans le faubourg avec leurs seuls bâtons. Ils firent face tant que leurs adversaires se battirent à l'arme blanche, mais lorsque

8. RGADA, f. 141 (PDSL), 1678, $n^{\circ} 154,1.52$.

9. Ibidem, f. 16 . 
ces derniers ouvrirent le feu, les mousquetaires s'enfuirent, laissant sur les lieux le cadavre d'un de leurs camarades. Leur chef Ivan Pazuhin fut capturé par les assaillants et le Russe mystérieux s'éclipsa ${ }^{10}$.

Ces événements alarmèrent sérieusement les autorités russes. Ce n'était pas la première fois que de regrettables incidents opposaient des étrangers aux mousquetaires dans leurs fonctions de maintien de l'ordre et de protection des officiers du tsar. En 1639, étant à la recherche de tabac dans les maisons des marchands anglais, ils se heurtèrent à une résistance ouverte ${ }^{11}$. Le 9 mai 1644, lors de la tentative de fuite du prince Waldemar, les affrontements nocturnes entre les Danois et les patrouilles du guet firent de nombreux blessés, l'un d'eux mortellement, parmi les mousquetaires ${ }^{12}$. Durant l'été 1647 , à Moscou, un sous-secrétaire, des employés subalternes et des mousquetaires, chargés de la prévention des incendies, étaient venus inspecter l'ancien comptoir des Anglais ; ceux-ci « les battirent et lancèrent leurs chiens sur eux $\gg^{13}$. Dans la nuit du 3 septembre 1651, le bojarin prince Aleksej Nikitič Trubeckoj ainsi que les mousquetaires qui l'accompagnaient pour « éteindre un incendie et assurer la sécurité », durent affronter le résident suédois Carl Pommering et sa suite. Pommering « sortit à cheval, il était ivre ; il dégaina son épée et voulut transpercer le bojarin prince Aleksej Nikitič Trubeckoj ainsi que les mousquetaires qui avançaient derrière lui, [...] il blessa trois d'entre eux, [...] et ses gens, ceux du résident, tout aussi meurtriers [...] commencèrent à se battre et voulurent les frapper de leur épée $»^{14}$. Enfin, en 1664, dans l'estuaire de la Dvina, le navire du Hollandais Anton Devis [De Wiese ?] fit volontairement chavirer la barque russe dans lequel se trouvaient des commis des bureaux accompagnés de six mousquetaires, chargés d'inspecter le vaisseau et d'enquêter « sur l'épidémie de peste $\gg^{15}$.

10. RGADA, f. 35, 1678, $n^{\circ}$ 238, « Delo ob učinivšemsja v dome anglijskogo poslannika Ivana Gebdona ubijstve... » [Affaire du meurtre commis dans la maison de l'envoyé anglais Ivan Hebdon...], 1. 89-90.

11. RIB, t. II, n 205, p. 944 : « Nakaznaja pamjat’ Matveju Zubovu ob učinenii rozyska po povodu proisšedših v Vologde vraždebnyh stolknovenij anglijskih torgovyh ljudej s russkimi » (1639, oktjabr' 18) [Instructions données à Matvej Zubov pour enquêter sur les heurts survenus à Vologda entre marchands anglais et Russes, 18 octobre 1639].

12. Aleksandr Golubcov, Prenija o vere, vyzvannye delom carevny Iriny Mihajlovny $i$ koroleviča Val'demara [Controverses sur la foi suscitées par [le mariage manqué] de la princesse Irina Mihajlovna avec le prince Waldemar], M., 1888, p. 153-154.

13. RGADA, f. $35,1678, n^{\circ} 238,1647, n^{\circ} 162$ « Delo po čelobitnoj anglijskogo agenta Spensora Britona o priezde na anglijskoe podvor'e strel'cov s ih načal'nikami i bit'e ego ljudej... » [Affaire de la plainte déposée par l'agent anglais Spencer Briton concernant l'intrusion dans le comptoir anglais de mousquetaires avec leurs officiers et des coups qu'ils ont donnés à ses gens...], 1. 4-9.

14. Gramota carja Alekseja Mihajloviča k koroleve Hristine ot 17 sentjabrja 1651 goda [Lettre du 17 septembre 1651, du tsar Aleksej Mihajlovič à la reine Christine], in Konstantin Jakubov, éd., Rossija i Švecija v pervoj polovine XVII V. [La Russie et la Suède dans la première moitié du XVII siècle], M., 1897, p. 390-391.

15. RGADA, f. 141, op. 3, 1664, $\mathrm{n}^{\circ}$ 198, « Čelobitnaja arhangel’skogo gorodničego Ivana Nekrasova i perevodčika Ftora Leont'eva s tovarišči o pričinenii ot priezžego torgovogo inozemca Antona Devisa i ego korabel’̌̌čika velikogo nasil’stva » [Plainte du commis de la 
Cependant, l'attaque d'une patrouille par des étrangers armés, la mise en déroute des mousquetaires, un meurtre et l'enlèvement d'un officier du tsar étaient sans précédent dans le faubourg des Étrangers. Sur l'ordre du tsar fut ouverte une enquête approfondie, dirigée par le panetier Semen Erofeevič Poltev et le secrétaire Fëdor Kuzmiščev. Les enquêteurs avaient pour mission de quadriller systématiquement, de porte à porte, le faubourg et d'y « interroger chacun séparément ${ }^{16}$. Le faubourg fut bouleversé de fond en comble. Près de cent dépositions figurent dans le dossier, signées par des Russes et des étrangers, dont certains très connus comme Patrick Gordon, Paul « Menezius » (Menzies), William Bruce.

L'enquête établit rapidement que les gens de Hebdon étaient à l'origine de l'attaque. Et « le grand souverain Fëdor Alekseevič, ayant entendu l'affaire, donna ordre et les bojare arrêtèrent de convoquer au secrétariat des Ambassades les gens de l'envoyé, pour y être interrogés sur le meurtre [...] et si l'envoyé refusait d'envoyer ses gens pour interrogatoire, de les faire venir de force. $\gg^{17}$.

Les Anglais inculpés ne nièrent pas leur participation à l'attaque mais affirmèrent qu'il s'agissait d'un tragique malentendu, car dans l'obscurité ils avaient soi-disant pris les mousquetaires pour des brigands. Pas un n'avoua le meurtre ou même avoir été porteur d'une arme à feu. Vaines également furent les tentatives des enquêteurs d'identifier le Russe.

Une telle dissimulation ne peut qu'éveiller, chez l'historien, des soupçons bien précis. De nombreux diplomates étrangers s'efforçaient, parfois non sans succès, de trouver un informateur clandestin au sein de l'administration russe. Grigorij Karpovič Kotošihin, sous-secrétaire au secrétariat des Ambassades, qui fournit en données confidentielles trois résidents suédois successifs, est le plus connu, mais certainement pas le seul de ces agents.

Les documents attestent que l'envoyé anglais John Hebdon disposait bel et bien d'un informateur de ce genre. Parmi les nombreuses plaintes déposées contre l'envoyé, la requête du colonel anglais John (Jagan) Cooper attire particulièrement l'attention. Cooper se plaignait de Hebdon, qui aurait dit « en présence de colonels et d'autres personnes dignes de confiance » que Cooper était « traître au roi d'Angleterre », et qu'il avait « rédigé et signé une note préconisant de ne pas rétablir les anciens privilèges accordés à Sa Royale Majesté, car les Anglais continueraient de toute façon à commercer avec la Russie, même en payant des droits de douane. Et l'envoyé a déclaré, devant des personnes dignes de confiance, avoir acheté cette note sur les privilèges, soi-disant signée de ma main, et la leur a montrée [...] et l'envoyé m'a injurié [...] et m'a traité de tous les noms, il voulait tuer mon fils à coups d'épée et a menacé de m'abattre, s'il me rencontrait quelque part ${ }^{18}$.

ville (gorodničij) Ivan Nekrasov, de ses adjoints et de l'interprète Secundus Leont'ev concernant les extrêmes violences commises par le marchand étranger Anton Devis et par le capitaine de son navire] f. 1-3.

16. RGADA, f. $35,1678, \mathrm{n}^{\circ} 238,1.25$.

17. RGADA, f. 141 (PDSL), 1678, n 154, 1. 84.

18. Ibidem, 1. 3 . 
La réaction des autorités russes ne se fit pas attendre. Le sous-secrétaire Maksim Burcov, du secrétariat des Ambassades, fut dépêché chez l'envoyé pour le sommer de remettre la note, de révéler l'identité du vendeur, et de s'expliquer au sujet des menaces adressées au colonel Cooper. L'envoyé refusa de livrer le document, ou le nom de son vendeur; en réponse aux plaintes de John Cooper, il demanda ce que « le colonel avait à voir avec lui, l'envoyé. Que Cooper expose donc cela à leur souverain à tous deux, à Sa Royale Majesté, mais ici, en tant qu'envoyé, il n'était pas tenu de fournir de réponse à la plainte du colonel ${ }^{19}$.

L'existence d'une telle note n'a rien en soi d'invraisemblable. Il arrivait en effet que les autorités moscovites s'adressent aux étrangers pour obtenir diverses informations, et les consultent sur des questions d'ordre juridique, économique, politique et militaire. En 1646, par exemple, des représentants du corps des marchands étrangers furent convoqués au secrétariat des Ambassades et interrogés sur la façon dont certaines questions juridiques «étaient traitées dans le droit des pays étrangers ». Spencer Briton intervint au nom des Anglais, Pieter De la Dalle au nom des Hollandais, Peter Marselis, au nom des Danois, et Heinrich Kellermann (Andrej Kelderman) au nom des marchands étrangers de Moscou $)^{20}$. La contribution qu'apporta Peter Marselis à l'élaboration du nouveau règlement de Commerce de $1667^{21}$ est bien connue. De même, le général Patrick Gordon, dans les années 1680 , conseillait régulièrement le « grand chancelier » Vasilij Vasil’evič Golicyn en matière politique et militaire et il établit à sa demande une note circonstanciée sur les perspectives d'une campagne en Crimée ${ }^{22}$.

Par ailleurs, le colonel Cooper niait avec véhémence être l'auteur de la note et pria le tsar d'informer par lettre le roi d'Angleterre « que l'envoyé [1]'avait, lui qui était innocent, déshonoré et couvert de honte ». Ainsi « en Angleterre, après sa mort, [lui], [ses] enfants et [sa] famille n'auraient pas à subir un opprobre et une honte éternels ». Il n'est pas exclu que Hebdon ait eu entre les mains un document falsifié, qu'il ait été fabriqué à sa demande ou à son insu. De plus, la signature du colonel pouvait aussi bien être imitée qu'authentique. Le commis indélicat avait en effet pu tirer parti du fait que cet étranger avait une piètre connaissance du russe pour lui faire signer un document, en le trompant sur son contenu. En 1662, l'Écossais

\section{Ibidem, 1. 4.}

20. RGADA, f. 141, op. 2, 1646, n² 20, « Doprosnye reči raznyh gostej inozemcev v uloženijah ih nemeckih pravah v sudnom kakom velikom dele dvum svideteljam veret' li » [Dépositions de divers marchands étrangers concernant les dispositions de leur droit, sur la question de savoir si deux témoins suffisent dans un procès important], 1. 1-2.

21. Konstantin Vasil’evič Bazilevič, « Novotorgovyj ustav 1667 g. (K voprosu o ego istočnikah) » [Le nouveau règlement de Commerce de 1667 (Contribution à une étude de ses sources)], Izvestija AN SSSR, VII serija, Otd. obščestvennyh nauk, n 7, L., 1932, p. 597-617 ; Andrej Vladimirovič Demkin, Zapadnoevropejskoe kupečestvo $v$ Rossii $v$ XVII $v$. [Les marchands d'Europe occidentale en Russie au XVII siècle], M. : Institut rossijskoj istorii RAN, 1994, fasc. 1, p. 42.

22. Aleksandr Gustavovič Brikner, Patrik Gordon i ego dnevnik [Patrick Gordon et son journal], SPb., 1878, p. 45-46. 
Patrick Gordon avait été victime d'une machination semblable, organisée par ses adversaires : il réussit à s'éviter de sérieux ennuis en subornant un autre commis ${ }^{23}$.

Le temps passait et les Anglais s'en tenaient fermement à leurs dépositions. L'envoyé protestait, insistant sur l'innocence des siens et exigeant leur libération immédiate. Il affirmait en outre que, même s'ils étaient coupables, leur statut diplomatique les protégeait de toute enquête criminelle.

Les autorités russes se hâtèrent de se renseigner à ce sujet. « Le colonel Paul Menezius, au cours d'une conversation » chez le bojarin Jurij Alekseevič Dolgorukov déclara que, lorsque « le factieux criminel » Olivier Cromwell gouvernait l'Angleterre, le frère de l'ambassadeur du Portugal à Londres se rendit coupable d'un meurtre : « il tua un homme d'un coup de pistolet » et fut décapité en conséquence $^{24}$. Menezius faisait ainsi preuve d'une remarquable subtilité diplomatique. Se trouvant dans une situation précaire après la chute et l'exil de son protecteur Artamon Sergeevič Matveev, l'officier écossais voulait en effet donner une réponse qui convienne aux autorités russes : ni le statut diplomatique, ni même la parenté avec l'ambassadeur n'avaient suffi à éviter le châtiment. Mais en rappelant que c'était là une décision du «factieux criminel »Cromwell, il insinuait que le souverain russe légitime, « frère bien-aimé » du roi d'Angleterre, ne pouvait guère s'inspirer de cet exemple.

Cependant, l'enquête était visiblement au point mort. Les Anglais étaient libérés au fur et à mesure et seul demeura en prison Karel Goosens (Colbert écrit Goossens ${ }^{25}$ ), l'étranger qui accompagnait le Russe, la nuit de l'altercation. Une fois identifié, il se révéla être une figure intéressante : originaire de Courlande, et non pas Anglais, c'est en Suède qu'il fut enrôlé par Hebdon, peu de temps avant de franchir la frontière russe. Sans aucun doute, le fait que Jan (Ivan) Goosens, le père de Karel, ait longtemps travaillé en tant que traducteur en Moscovie joua un rôle important dans le choix du diplomate ${ }^{26}$. La collaboration des Goosens père et fils représentait en effet pour l'envoyé un atout inestimable. Jan Goosens résidait depuis longtemps en Russie, puisque les premiers documents où son nom apparaît datent du règne du tsar Mihail Fëdorovič ${ }^{27}$. Goosens ne faisait pas partie du personnel du secrétariat

23. Patrick Gordon, « Dnevnik Patrika Gordona » [Le journal de Patrick Gordon], Čtenia V imperatorskom Obščestve istorii i drevnostej rossijskih, 1892 (3), p. 12-13, 21-22 (paraphrase détaillée de l'original). Dans l'édition complète du journal de Gordon, traduite en russe et annotée par Dmitrij Gennad'evič Fedosov, en cours de publication (Patrik Gordon, Dnevnik 1635-1659, Dnevnik 1659-1667, Dnevnik 1677-1678, M. : Nauka, 2001, 2002, 2005), les passages correspondants se trouvent dans Dnevnik 1659-1667, p. 109-110 et 117.

24. RGADA, f. 141 (PDSL), 1678, $\mathrm{n}^{\circ} 154,1.6$.

25. Pierre Clément, éd., Jean-Baptiste Colbert, Lettres, instructions et mémoires..., vol. II/2, P., 1863, p. 605, note (NdT).

26. RGADA, f. $35,1678, n^{\circ} 238,1.129-130$.

27. Materialy $k$ istorii mediciny [Matériaux pour servir à une histoire de la médecine], vol. I, SPb., 1881, p. 396-397 : «Čelobitnaja doktora Vendelina Sibilista o dozvolenii iskat’ po sudu v Aptekarskom prikaze besčest ’ja na kurljandce Ivane Gossene » (26 dekabrja 1644 g.) [Requête du docteur Wendelin Sibilist demandant l'autorisation de poursuivre le Courlandais Jan Goosens devant le secrétariat des Apothicaires, pour atteinte portée à son honneur, 26 décembre 1644]. 
des Ambassades, mais on faisait parfois appel à lui pour certaines missions diplomatiques. En 1668, notamment, il accompagna l'envoyé Petr Ivanovič Potëmkin et le secrétaire Semen Vladimirovič Rumjancev en France. Il y fut surpris en flagrant délit de déloyauté par les diplomates russes et fut même, en une autre occasion, roué de coups par l'envoyé et mis aux arrêts quelques jours parce que soupçonné de trahison ${ }^{28}$.

Durant les interrogatoires, Karel Goosens s'en tenait fermement à la même ligne de défense que les autres gens de Hebdon. Cela dura jusqu'à la fin du mois de mars. Cependant, le diplomate s'était mis à préparer en toute hâte son départ de Russie. Quand Goosens apprit que son employeur s'apprêtait à quitter le pays sans avoir obtenu sa libération, il fut certainement bouleversé. Il se mit donc à collaborer activement à l'enquête, fournit des témoignages détaillés sur les porteurs d'armes à feu au moment de l'agression, susceptibles par conséquent d'avoir tué le mousquetaire $^{29}$. Avec l'aide de Goosens, les enquêteurs parvinrent à établir l'identité du principal suspect du meurtre : un certain Charles Jordon (ou : Jordan ; « Karlus Gerdon »).

Lors de la confrontation, Jordon « [...] nia tout d'abord, puis, s'approchant du secrétaire du Conseil, Larion Ivanovič [Ivanov], il finit par reconnaître son crime : il avoua que lui, Karl, était coupable de meurtre devant Dieu le Père [...] et devant Sa Majesté le tsar. Il tomba à genoux et se mit à pleurer et, en pleurs, il dit [...] [que lui,] Karl avait un pistolet armé à la main et que soudain, il ne sait comment, le rouet du pistolet se lâcha ${ }^{30}$ et le coup de feu partit. Il ne visait personne [...] il causa la mort sans préméditation, involontairement. » Jordon ajouta que le diplomate ne savait pas que les auteurs du meurtre étaient des gens à lui, dans la mesure où « [...] ils n'avaient rien avoué à l'envoyé et affirmé qu'aucun d'eux n'avait tiré, de peur que l'ambassadeur ne les châtie. ${ }^{31}$

Les aveux pittoresques de Jordon donnent l'impression d'être parfaitement pensés et orchestrés, de manière à minimiser les conséquences fâcheuses pour Jordon lui-même et pour Hebdon. Le corps d'Andrej Nesterov, le mousquetaire tué, fut examiné : «Andrjuška avait reçu deux balles dans le dos » ${ }^{32}$. Les assurances de Jordon sur le caractère isolé et fortuit du coup de feu paraissent donc très peu

28. «Statejnyj spisok P.I. Potemkina » [Rapport de Petr Ivanovič Potemkin »], in Putešestvija russkih posloV XVI-XVII VV. [Les voyages des ambassadeurs russes auX XVI ${ }^{\mathrm{e}}$-XVII ${ }^{\mathrm{e}}$ siècles], M.-L., 1954, p. 238, 431 ; "Journal du sieur de Catheux », RNB, inv. 312, franç. F. IV, n 155 , 1668, f. 22 ; Vasilij Rudol'fovič Novoselov, « Russkie posly vo Francii v 1668 g. Dva otčeta ob odnoj missii » [Les ambassadeurs russes en France en 1668. Deux rapports d'une même mission], in Inozemcy $v$ Rossii $X V-X V I I V V$. [Les étrangers en Russie auX $X V^{\mathrm{e}}-X V I I^{\mathrm{e}}$ siècles], M. : Zapad-Vostok, 2006, p. 346-355, ici p. 353-354.

29. RGADA, f. $35,1678, \mathrm{n}^{\circ} 238,1.165-167$.

30. «ROÜET, se dit aussi d'une petite rouë d'acier qu'on applique sur la platine d'une arquebuse, d'un pistolet, ou autre arme à feu, qu'on bande avec une clef, \& qui en se laschant avec violence, fait du feu par le moyen d'une pierre qu'on trouve dans les mines de cuivre » (Dictionnaire universel d'Antoine Furetière, 1688-1689).

31. RGADA, f. $35,1678, \mathrm{n}^{\circ} 238,1.180-182$.

32. RGADA, f. 141 (PDSL), 1678, $\mathrm{n}^{\circ} 154,1.69$. 
convaincantes. De même, l'ignorance de son employeur au sujet du meurtre. En effet, lorsque les Anglais avaient amené Ivan Pazuhin chez Pieter Hasenius, John Hebdon en personne était sorti pour l'interroger. Lorsqu'il sut qui était son prisonnier, le diplomate tenta sans désemparer d'étouffer l'affaire : « [II] ordonna à Ivan de n'informer le souverain ni de l'altercation ni du meurtre du mousquetaire [c'est moi qui souligne, S. O.], mais de venir le voir à l'Hôtel des Ambassadeurs, afin qu'il lui accorde sa protection et éclaircisse ce crime $»{ }^{33}$

Cette affaire, qui s'éternisait, embarrassait les autorités moscovites. Le problème le plus difficile était : « que faire du meurtrier ? » L'exécuter, comme le voulait la loi, signifiait détériorer les relations anglo-russes, déjà médiocres. Lui accorder l'impunité aurait porté atteinte au prestige des autorités. Une solution fut finalement trouvée.

Le 12 mars 1678, Charles Jordon fut mené sur la place Rouge, « où ont lieu les exécutions, face à l'allée des selliers », et on lui lut la sentence : «[...] tu es condamné à mort pour ce crime et pour meurtre ». Puis on devait, conformément aux instructions, mettre la tête du condamné sur le billot, ensuite le relever et annoncer : «Le souverain Fëdor Mihajlovič, en souvenir de son père [...] le souverain Aleksej Mihajlovič de glorieuse mémoire, te gracie et donne l'ordre de te laisser quitter Moscou avec l'envoyé $»^{34}$. Hebdon quitta Moscou précipitamment au mois de mars. Aussitôt après, le tsar Fëdor Alekseevič adressa au roi Charles II une lettre furieuse, disant que l'envoyé avait été à l'origine « de nombreux actes indignes et contraires aux usages diplomatiques, et d'un meurtre », et exigeant en outre qu'il fût puni « afin qu'à l'avenir, d'autres n'osent pas agir de même ni semer la discorde entre nous, souverains $»^{35}$.

Certaines questions demeurent. Que souhaitait obtenir Hebdon durant son séjour d'une année dans la capitale russe ? Qui était ce mystérieux Russe, que les Anglais réussirent à reprendre aux mousquetaires ? Et pourquoi le diplomate prit-il des risques aussi énormes?

La mission de John Hebdon fils différait sensiblement des tentatives de ses prédécesseurs pour rétablir les privilèges commerciaux anglais en Russie. John Hebdon père, lorsqu'il était envoyé extraordinaire, et avant lui, Charles Howard, comte de Carlisle, en qualité d'ambassadeur, avaient réclamé de façon insistante et intransigeante leur rétablissement. John Hebdon fils, en revanche, se vit offrir un compromis. Puisque les autorités russes refusaient catégoriquement le retour au régime d'avant 1649, à savoir l'entrée en franchise des marchandises anglaises, John Hebdon fils accepta que les Anglais paient des droits de douane, demandant seulement que ceux-ci soient calculés « à l'ancienne », c'est-à-dire inférieurs à ceux prévus par le nouveau règlement de Commerce de $1667^{36}$.

33. Ibidem, 1. 68.

34. RGADA, f. $35,1678, n^{\circ} 238,1.192$.

35. Ibidem, 1. 208-210.

36. RGADA, f. $35,1676, \mathrm{n}^{\circ} 234,1.27-29,205$. 
Le gouvernement anglais avait probablement placé de grands espoirs dans la mission de Hebdon fils, pensant que l'avènement du nouveau souverain Fëdor Alekseevič et le changement de direction au secrétariat des Ambassades permettraient de faire avancer la question particulièrement sensible des privilèges commerciaux, qui était au point mort. John Hebdon fils comprenait fort bien que, dans ces conditions, un fiasco total pouvait avoir des conséquences désastreuses sur sa carrière future. On peut donc supposer que l'ambition de l'envoyé extraordinaire a joué un rôle non négligeable dans cette affaire. Confronté à un gouvernement russe inflexible, il fit l'impossible pour se réhabiliter, au moins partiellement. Visiblement, Hebdon s'efforçait d'obtenir des preuves tangibles montrant que son entreprise avait échoué à cause d'intrigues fomentées par des « traîtres » et reporter ainsi sur eux une partie de la responsabilité de son échec.

La note du colonel Cooper obtenue par l'envoyé devait servir de preuve qu'il existait à la cour de Russie un parti secrètement hostile au roi d'Angleterre, qui avait fait échouer la mission de Hebdon. Celui-ci recherchait sans doute d'autres preuves, mais il lui fallait pour cela un complice au sein de l'administration moscovite. Les circonstances ont fait qu'après sa rencontre avec le diplomate dans le faubourg des Étrangers, l'agent russe fut arrêté par les mousquetaires. Visiblement, Hebdon redoutait moins un scandale en Russie qu'un retour sans gloire en Angleterre, ou, pis encore, que le gouvernement anglais découvre ses peu reluisantes activités. Dans ces conditions, Hebdon n'avait d'autre choix que de donner l'ordre à ses gens d'attaquer les mousquetaires.

L'épisode pittoresque de la mission de John Hebdon à Moscou illustre, d'une façon qui n'a rien de conventionnel, l'histoire des relations anglo-russes au cours de la seconde moitié du XVII ${ }^{\mathrm{e}}$ siècle, et particulièrement la question épineuse entre toutes des privilèges commerciaux.

Musée du Kremlin de Moscou

Orlen@yandex.ru 